\title{
Development of Hodgkin's disease in the course of liver cirrhosis and impaired monocyte function
}

\author{
MICHAEL DAN \\ M.D. \\ MEDINA JEDWAB \\ M.D.
}

\author{
AVI HASSNER \\ M.D.
}

SHLOMO SHIBOLET

M.D.

Department of Internal Medicine D, Ichilov Hospital and University of Tel Aviv, Sackler School of Medicine, Tel Aviv, Israel

\begin{abstract}
Summary
The monocyte function, as measured by phagocytosis and killing of Candida albicans and Candida pseudotropicalis by peripheral blood monocytes, was found impaired in a patient with cirrhosis of the liver on two separate determinations before the occurrence of bleeding from oesophageal varices. Unexpectedly, Hodgkin's disease was diagnosed in enlarged abdominal lymph nodes found on the occasion of an emergency portocaval shunt operation.
\end{abstract}

KEY WORDS: bacterial peritonitis, phagocytosis.

\section{Introduction}

The development of a lymphoproliferative disease in association with a chronic liver disease, although uncommon, has been documented in several reports (Heimann, 1971; Viteri, Vernace and Schaffner, 1976; Slavin et al., 1974). It was suggested that this association is possibly not a fortuitous coincidence, but influenced by the prolonged antigenic stimulation as a result of the impaired reticuloendothelial function in the diseased liver (Naparstek and Eliakim, 1979). In contrast to earlier published opinions (Sherlock, 1977) we found distinct decreased monocyte function in this case, as in other cirrhotic patients (Hassner et al., 1979).

\section{Case report}

Cirrhosis of the liver was diagnosed in a 57-yearold man in February 1978 following the appearance of ascites and peripheral oedema. There was no history of alcohol abuse, infectious hepatitis or administration of hepatotoxic drugs. The diagnosis of liver cirrhosis was confirmed by liver biopsy. He received diuretic therapy which was continued after discharge.
In December 1978 the patient was admitted to our $\stackrel{+}{\sim}$ department with fever and abdominal pains shown to $ᄋ$

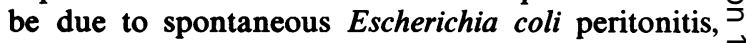
which responded to antibiotics therapy. He recovered and was discharged after 21 days with diuretic therapy, and appeared regularly for follow-up.

The patient was readmitted in August 1979 with $\stackrel{\odot}{\varnothing}$ another episode of chills, high fever and abdominal ${ }^{+}$ pains. Abdominocentesis revealed turbid fluid rich in polymorphonuclear cells, and antibiotic therapy was initiated. On the second hospital day bleeding from $\bar{\partial}$ oesophageal varices occurred, and a Blakemore tube $\stackrel{\curvearrowright}{\%}$

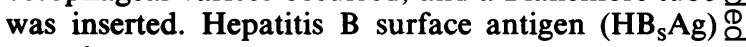
was absent.

Sixteen days later he had melaena. After demon- $\frac{0}{3}$ stration of oesophageal varices on gastroscopy, an end-to-end portocaval shunt was performed. At laparatomy enlarged para-aortic lymph nodes were found. Histological examination of the enlarged 3 lymph nodes revealed Hodgkin's disease, mixed cellularity type. Reed-Sternberg cells were found also in the bone marrow. Because of the poor prognosis 8 due to advanced liver disease, we decided not to treat $₹$ the lymphoproliferative disease. He succumbed 6 윽 months later from hepatic coma.

At autopsy generalized lymphoadenopathy was found, predominantly para-aortic, coeliac and mesenteric. The abdomen contained $250 \mathrm{ml}$ of ascites. $\%$ The liver was contracted and diffusely nodular, and $N$ the spleen weighed $850 \mathrm{~g}$.

\section{Special study}

Monocyte activity was evaluated during a clinical $T$ study of monocyte function in patients with cirrhosis of the liver.

Monocyte phagocytosis and intracellular killing of $\frac{\vec{\Phi}}{2}$ 
TABLE 1. Peripheral monocyte activity in the present patient compared to a group of cirrhotic patients and normal controls

\begin{tabular}{|c|c|c|c|c|}
\hline & \multicolumn{2}{|c|}{ Phagocytosis* } & \multicolumn{2}{|c|}{ Intracellular killing $\dagger$} \\
\hline & C. pseudotropicalis & C. albicans & C. pseudotropicalis & C. albicans \\
\hline This case & $\begin{array}{l}1.2 \pm 0.02 \\
(P<0.05)\end{array}$ & $\begin{array}{c}1.23 \pm 0.02 \\
(P<0.05)\end{array}$ & $\begin{array}{c}24 \cdot 6 \pm 2 \cdot 6 \\
(P<0 \cdot 0025)\end{array}$ & $\begin{array}{c}21.8 \pm 2.96 \\
(P<0.0005)\end{array}$ \\
\hline $\begin{array}{l}\text { Cirrhotic patients } \\
(n=16) \\
\text { Controls } \\
(n=9)\end{array}$ & $\begin{array}{c}1 \cdot 17 \pm 0.08 \\
(P<0.0025) \\
1.25 \pm 0.06\end{array}$ & $\begin{array}{l}1.2 \pm 0.07 \\
(P<0.005) \\
1.33 \pm 0.13\end{array}$ & $\begin{array}{c}24.99 \pm 7 \cdot 41 \\
(P<0 \cdot 0001) \\
40 \pm 9 \cdot 13\end{array}$ & $\begin{array}{l}28 \cdot 22 \pm 4 \cdot 11 \\
(P<0 \cdot 0001) \\
44 \cdot 22 \pm 6 \cdot 17\end{array}$ \\
\hline
\end{tabular}

${ }^{*}$ Mean of yeast cells per monocyte; †Mean $\%$ of killed yeasts. Values are means \pm s.d. In parentheses are $\boldsymbol{P}$ values comparing results from the present patient and a group of cirrhotic patients to controls. Data about controls and cirrhotic patients obtained with permission (Hassner et al., 1979).

Candida albicans and Candida pseudotropicalis was measured using the method of Territo and Cline (1977). The measurements were performed under the same basic conditions in two blood samples at 2monthly interval, 1 year before the portocaval shunt operation. The results are compared to normal controls matched for age and ethnic origin. Statistical analysis was by Student's $t$-test.

Table 1 gives results obtained from nine normal controls, 16 patients with cirrhosis of the liver and the present case.

We found that phagocytosis and intracellular killing of Candida yeasts by peripheral blood monocytes from our patient were statistically significantly reduced compared to a control group, and were in accord with results obtained in a previous study of patients with cirrhosis of the liver (Hassner et al., 1979).

\section{Discussion}

The causal relationship between chronic liver disease and lymphoproliferative disease is difficult to prove, and only a large prospective study will answer this question clearly. However, several mechanisms explaining the development of lymphoma in the course of liver disease have been offered: prolonged antigenic stimulation (Prytz et al., 1977), immunosuppressive therapy (Viteri et al., 1976; Harris, 1976) and presence of hepatitis B surface antigen (Wands $e t$ al., 1975).

Prolonged antigenic stimulation has been suggested to be operative in chronic liver disease, resulting most probably from the decreased antigenic elimination by the diseased liver with impaired reticuloendothelial function (Prytz et al., 1977). The permanent antigenic stimulation maintains a proliferation of the lymphoid system which could deviate towards malignancy (Editorial, 1970). This mechanism was demonstrated experimentally in animals (Metcalf, 1961), and was offered to explain also the increased frequency of lymphomas in autoimmune disease (Miller, 1967).
The reticuloendothelial system, or more properly designated, the mononuclear phagocyte system, has been widely studied in recent years (Cline, 1978). The activity of Kupffer cells, which belong to this system, has already been partially studied in the diseased liver, and was found impaired (Prytz et al., 1977; Bjorneboe, Prytz and Orskov, 1972). Sherlock (1977) has stated that there were no data available that monocyte function is impaired in cirrhosis of liver, but that these cells are functionally incapacitated by the by-pass of portal blood by way of the portocaval anastomoses. In contrast, recent preliminary publications have reported impaired monocyte function in liver cirrhosis as measured by phagocytosis or killing of Candida (Hassner et al., 1979) or by measuring monocyte chemotaxis (Holdstock, Hill and Wright, 1979).

While in the majority of reported cases of the occurrence of lymphomas in chronic liver disease immunosuppressive therapy was given (Viteri et al., 1976; Pirrote, 1974), it is of note that no such treatment was administered in our case. In this context the finding of impaired monocyte function in our patient deserves special attention.

Our findings of impaired monocyte function in patients with liver cirrhosis could point to an additional factor involved in the lowered resistance to infection of these patients. Furthermore, impaired monocyte function would most probably expose these patients to prolonged antigenic stimulation. The patient described in this report demonstrated two possible complications of the decreased immunocompetence in cirrhosis of the liver: the appearance of spontaneous recurrent peritonitis and the development of Hodgkin's disease in the abdominal lymph nodes. It seems that chronic liver disease should be added to the list of conditions with immunological disturbances associated with malignant diseases.

\section{References}

Bjorneboe, M., Prytz, H. \& Orskov, F. (1972) Antibodies to intestinal microbes in serum of patients with cirrhosis of the liver. Lancet, i, 58. 
CLINE, M.J. (moderator) (1978) Monocytes and macrophages: function and diseases. Annals of Internal Medicine, 88, 78.

EDITORIAL (1970) Lymphoid stimulation and lymphoid neoplasia. Lancet, ii, 596.

HARRIS, C. (1976) The carcinogenicity of anticancer drugs: A hazard in man. Cancer, 37, 1014.

Hassner, A., Kleter, Y., Jedwab, M., Aronson, M. \& Shibolet, S. (1979) Impaired monocyte function in liver cirrhosis. Lancet, i, 329.

HeimanN, R. (1971) Cirrhoses et lymphopaties malignes. Acta gastro-enterologica Belgicà, 34, 663.

Holdstock, G.E., HILl, S. \& WRIGHT, R. (1979) Peripheral monocytes in chronic liver disease. Gastroenterology, 77, A17.

Metcalf, D. (1961), Reticular tumours in mice subjected to prolonged antigenic stimulation. British Journal of Cancer, 15, 769.

MILLER, D.G. (1967) The association of immune disease and malignant lymphoma. Annals of Internal Medicine, 66, 507.

NAPARSTEK, Y. \& ElIAKIM, M. (1979) Lymphoproliferative diseases and liver diseases. Harefuah, 96, 205.

PIRROTE, J.H. (1974) Development of Hodgkin's disease in the course of active hepatitis treated by immunosuppresive drugs. American Journal of Gastroenterology, 6, 230.

Prytz, H., Bjorneboe, M., Christofersen, P., Poulsen, H. \& $\subseteq$ ORSKov, F. (1977) Correlation between hepatic morphology and $\vec{F}$ immunoglobulins and antibodies to E. coli in cirrhosis. Gut, 18, 28.

SHERLOCK, S. (1977) Immunological changes in liver disease. Proceedings of the Royal Society of Medicine, 70, 851.

Slavin, S., Zlotnick, A., LeviJ, I.C. \& Eliakim, M. (1974) Clinical implications of monoclonal gammopathy in chronic liver disease. American Journal of Digestive Diseases, 19, 223.

TerRito, M.C. \& Cline, M.J. (1977) Monocyte function in man. Immunology, 118, 187.

Viteri, A., Vernace, S.S., \& ShaffNer, F. (1976) Extrahepatic $\overrightarrow{0}$ malignancy in chronic liver disease: report of six cases. Gastroenterology, 71, 1075

WANDS, J.R., ChURA, M.C., Roll, F.J. \& MADDRey, W.C. (1975) Serial studies of hepatitis associated antigen and antibody in patients receiving anti-tumor chemotherapy for myeloproliferative and lymphoproliferative disorders. Gastroenterology, 68, 105.

(Accepted 25 May 1983) 